OPEN ACCESS

Edited by:

Fabian Cieplik,

University Medical Center

Regensburg, Germany

Reviewed by:

Joanna Nakonieczna,

Intercollegiate Faculty

of Biotechnology of University

of Gdańsk and Medical University

of Gdańsk, Poland

Michaela Wenzel,

Chalmers University of Technology,

Sweden

*Correspondence:

Rikke H. Olsen

cava@sund.ku.dk

Specialty section:

This article was submitted to

Antimicrobials, Resistance

and Chemotherapy,

a section of the journal

Frontiers in Microbiology

Received: 29 September 2021 Accepted: 25 November 2021

Published: 05 January 2022

Citation:

Ronco T, Kappel LH, Aragao MF,

Biagi N, Svenningsen $S$,

Christensen JB, Permin A, Saaby L,

Holmstrøm K, Klitgaard JK, Sabat AJ,

Akkerboom V, Monaco M, Tinelli M,

Friedrich $A W$, Jana $B$ and Olsen $R H$

(2022) Insight Into the

Anti-staphylococcal Activity of JBC

1847 at Sub-Inhibitory Concentration.

Front. Microbiol. 12:786173.

doi: 10.3389/fmicb.2021.786173

\section{Insight Into the Anti-staphylococcal Activity of JBC 1847 at Sub-Inhibitory Concentration}

\author{
Troels Ronco' ${ }^{1}$, Line H. Kappel' ${ }^{2}$, Maria F. Aragao', Niccolo Biagi', Søren Svenningsen ${ }^{3}$, \\ Jørn B. Christensen ${ }^{3}$, Anders Permin ${ }^{4}$, Lasse Saaby ${ }^{5}$, Kim Holmstrøm ${ }^{5}$, \\ Janne K. Klitgaard ${ }^{2,6}$, Artur J. Sabat ${ }^{7}$, Viktoria Akkerboom ${ }^{7}$, Monica Monaco ${ }^{8}$, \\ Marco Tinelli ${ }^{9}$, Alexander W. Friedrich ${ }^{7}$, Bimal Jana ${ }^{10}$ and Rikke H. Olsen ${ }^{1 *}$ \\ ${ }^{1}$ Department of Veterinary and Animal Sciences, Faculty of Health and Medical Sciences, University of Copenhagen, \\ Copenhagen, Denmark, ${ }^{2}$ Research Unit of Molecular Microbiology, Department of Biochemistry and Molecular Biology, \\ University of Southern Denmark, Odense, Denmark, ${ }^{3}$ Department of Chemistry, Faculty of Science, University \\ of Copenhagen, Copenhagen, Denmark, ${ }^{4}$ Unibrains, Virum, Denmark, ${ }^{5}$ Bioneer A/S, Hørsholm, Denmark, ${ }^{6}$ Research \\ Unit of Clinical Microbiology, Institute of Clinical Research, University of Southern Denmark, Odense, Denmark, ${ }^{7}$ Department \\ of Medical Microbiology, University Medical Center Groningen, University of Groningen, Groningen, Netherlands, \\ ${ }^{8}$ Department of Infectious Diseases, Istituto Superiore di Sanità, Rome, Italy, ${ }^{9}$ Division of Infectious and Tropical Diseases, \\ Hospital of Lodi, Lodi, Italy, ${ }^{10}$ Department of Biology, Boston College, Chestnut Hill, MA, United States
}

Multidrug-resistant pathogens constitute a serious global issue and, therefore, novel antimicrobials with new modes of action are urgently needed. Here, we investigated the effect of a phenothiazine derivative (JBC 1847) with high antimicrobial activity on Staphylococcus aureus, using a wide range of in vitro assays, flow cytometry, and RNA transcriptomics. The flow cytometry results showed that JBC 1847 rapidly caused depolarization of the cell membrane, while the macromolecule synthesis inhibition assay showed that the synthesis rates of DNA, RNA, cell wall, and proteins, respectively, were strongly decreased. Transcriptome analysis of $S$. aureus exposed to sub-inhibitory concentrations of JBC 1847 identified a total of 78 downregulated genes, whereas not a single gene was found to be significantly upregulated. Most importantly, there was downregulation of genes involved in adenosintrifosfat (ATP)-dependent pathways, including histidine biosynthesis, which is likely to correlate with the observed lower level of intracellular ATP in JBC 1847-treated cells. Furthermore, we showed that JBC 1847 is bactericidal against both exponentially growing cells and cells in a stationary growth phase. In conclusion, our results showed that the antimicrobial properties of JBC 1847 were primarily caused by depolarization of the cell membrane resulting in dissipation of the proton motive force (PMF), whereby many essential bacterial processes are affected. JBC 1847 resulted in lowered intracellular levels of ATP followed by decreased macromolecule synthesis rate and downregulation of genes essential for the amino acid metabolism in S. aureus. Bacterial compensatory mechanisms for this proposed multitarget activity of JBC 1847 seem to be limited based on the observed very low frequency of resistance toward the compound.

Keywords: phenothiazine derivative, antimicrobial resistance, mode of action, RNA transcriptomics, Grampositive bacteria 


\section{INTRODUCTION}

Infections caused by multidrug-resistant pathogens constitute a worrisome one-health issue and, consequently, there is a need for novel clinical treatment solutions (Price et al., 2012; Cassini et al., 2019; Singer et al., 2019). The pipeline for novel antimicrobial seems to be drying out, despite an urgent need (Kmietowicz, 2017; Singer et al., 2019). As an alternative to a novel antimicrobial, there has been focus on identifying compounds to "re-sensitize" antimicrobial resistant bacteria to current available antibiotics. Compounds belonging to the group of phenothiazines have received particular attention for the properties to act synergistically with oxacillin against methicillin-resistant Staphylococcus aureus (MRSA) (Klitgaard et al., 2008), although the antimicrobial activity of phenothiazines as single-acting antimicrobials is limited, with minimal inhibitory concentration (MIC) values between 16 and $512 \mathrm{mg} / \mathrm{L}$, depending on the specific phenothiazine and strains under evaluation (Kristiansen et al., 2007; Nehme et al., 2018). Unfortunately, the toxicity of phenothiazines has limited their clinical relevance, e.g., because they readily pass the bloodbrain barrier (BBB) causing behavioral changes (Stenger et al., 2017). To hinder the latter, we have recently synthesized a phenothiazine (promazine) derivative, JBC 1847, that does not tend to pass the BBB. This derivative is a positively charged cationic amphiphilic compound containing a quaternary ammonium ion with a monoterpene side chain, which exhibits significantly improved antimicrobial activity compared with its "mother" compound (Ronco et al., 2020). It does not, however, show synergy with beta-lactams or other antimicrobials, which has led to the hypothesis that the antimicrobial mode of action of JBC 1847 is different from original phenothiazines (Ronco et al., 2020).

Previously, we have shown that JBC 1847 is superior to fusidic acid ( $2 \%$ topical crème) in reducing the bacterial load of MRSA in a murine MRSA wound model (Ronco et al., 2020), displays low eukaryotic cytotoxicity (Ronco et al., 2021a), and that the compound is active against Grampositive bacteria embedded in biofilm (Ronco et al., 2021b). The latter is particularly important as biofilms constitute a major clinical challenge due to lack of penetrance of most antibiotics through the viscous biofilm matrix (Sharma et al., 2019) and/or the lack of targeting bacteria in a more slowly growing state as often observed in biofilm (Anderl et al., 2003). The aim of the present study was therefore to investigate the effect of JBC 1847 on S. aureus and to determine the frequency of resistance development in $S$. aureus and Enterococcus faecium.

\section{MATERIALS AND METHODS}

\section{Bacterial Strain and Antimicrobial Compounds}

Staphylococcus aureus JE2, a plasmid-cured derivative of a MRSA isolate USA300 (Fey et al., 2013), was used as reference strain to study the effect of JBC 1847 on S. aureus. The isolate was stored in brain-heart infusion (BHI) broth with $15 \%$ glycerol at $-80^{\circ} \mathrm{C}$ until used.

For determination of the frequency of resistance, two additional isolates were included: S. aureus CC398 and E. faecium ATCC 700221. As JBC 1847 and $\mathrm{Ca}^{++}$-activated daptomycin (DAP) are both positively charged (which likely contributes to the antimicrobial activity of the latter), it was investigated if JBC 1847 displays cross-resistance with DAP. For this purpose, a DAP sensitive wild-type strain (IT1-S) and its DAP-resistant variant (IT4-R) (Sabat et al., 2018) were applied.

The antimicrobial compounds JBC 1847 and T5 (molecular structures are presented in Supplementary Figure 1) were synthesized at University of Copenhagen, Denmark, as previously reported (Jørgensen et al., 2020; Ronco et al., 2020). All other antimicrobials were purchased at Sigma-Aldrich (Brøndby, Denmark), unless mentioned otherwise.

\section{Determination of the Antimicrobial Activity}

The broth dilution method based on the guidelines from Clinical and Laboratory Standards Institute was applied for the determination of the MIC and the minimal bactericidal concentration $(\mathrm{MBC})$ values. MIC values were determined in 96-well plates as previously described by Ronco et al. (2020). To determine MBC, $20 \mu \mathrm{l}$ from each well in the 96-well plate from the MIC determination were inoculated on blood agar (BA) plates (blood agar base, Oxoid, Roskilde, Denmark; supplemented with $5 \%$ bovine blood), incubated at $37^{\circ} \mathrm{C}$ for $18-24 \mathrm{~h}$ and colonies were then enumerated. MBC was determined as the concentration reducing the initial bacterial concentration per well with $\geq 99.9 \%$. MBC determination for cells in stationary phase was done as follows: After 24-h incubation of $S$. aureus JE2 at $37^{\circ} \mathrm{C}$ in Müller Hinton broth (Oxoid), the MH broth was diluted 1:4 in $0.9 \%$ saline (final volume $5 \mathrm{ml}$ ) and mixed with JBC 1847 to yield concentrations between 0.25 and $8 \mathrm{mg} / \mathrm{L}$ of $\mathrm{JBC}$ 1847. A negative control (broth without JBC 1847) was also included. All tubes were incubated at $37^{\circ} \mathrm{C}$ for $24 \mathrm{~h}$, and the CFU per milliliter of each tube was enumerated. The MBC was determined as the lowest concentration yielding a $99.9 \%$ reduction of the CFU in the negative control. The experiment was repeated twice.

Determination of the JBC 1847 MIC in a DAP-sensitive strain of $S$. aureus and a DAP-resistant strain, respectively, were done according to Sabat et al. (2018).

\section{Determination of Single-Step Resistance Generation Mutations}

The frequencies of single-step spontaneous mutations in five bacterial strains were determined. Log-phase bacteria at a concentration of $10^{6}-10^{10} \mathrm{CFU} / \mathrm{ml}$ were plated onto $\mathrm{BHI}$ agar plates containing antimicrobial compounds in a concentration of $8 \times$ MIC for each compound, and the plates were incubated at $37^{\circ} \mathrm{C}$ for $48 \mathrm{~h}$. In addition, several dilutions of each culture were plated on drug-free media to provide accurate colony counts. Resistance frequencies were calculated by dividing the 
number of colonies growing on antibiotic plates by the total number of CFU plated as outlined by Butler et al. (2002). Each experiment was performed in triplicate on separate days, and mutation frequencies represent average values. To verify the stability of each resistant organism, the organisms were transferred several times in drug-free medium and medium containing a drug concentration of $4 \times \mathrm{MIC}$ and were again tested for resistance.

\section{Assessment of Membrane Potential Using Flow Cytometry}

Measurement of the membrane potential using the BacLight Bacterial Membrane Potential Kit (Invitrogen) in three biological replicates was done according to Wassmann et al. (2018). Briefly, an overnight culture of $S$. aureus JE2 was diluted to an $\mathrm{OD}_{600} 0.02$ in $\mathrm{BHI}$ and incubated at $37^{\circ} \mathrm{C}$ until early exponential phase $\left(\mathrm{OD}_{600} 0.3\right)$. The culture was then diluted 1:100 in sterile phosphate-buffered saline and split into separate tubes containing $1 \mathrm{ml}$ diluted culture each. One sample was treated with $10 \mu \mathrm{M}$ of CCCP (carbonyl cyanide 3chlorophenylhydrazone) to depolarize the cells, thereby acting as a depolarized control; one sample was treated with DMSO, while four samples were treated with four different concentrations of JBC 1847 (0.0625-0.50 mg/L). All samples were stained for 1530 min with $10 \mu \mathrm{M}$ of DiOC2 dye and the last sample was left as an unstained control. After staining, the samples were analyzed in a BD FACS Aria II flow cytometer (Becton, Dickinson and Company, Franklin Lakes, NJ, United States). Hereafter, $10^{4}$ events were analyzed, while exciting the particles with a laser $(488 \mathrm{~nm})$ and collecting the green and red fluorescence.

\section{Isolation of Total Cellular RNA}

Here, the RNA expression response of $S$. aureus JE2 to subinhibitory concentrations of JBC 1847 was investigated relative to the untreated control. For comparative reasons, the responses of S. aureus JE2 to sub-inhibitory concentrations of promazine, the "mother" compound of JBC 1847 and to T5, and another phenothiazine derivative with structural similarity to JBC1847 (Jørgensen et al., 2020), were also included in the analysis. Initially, growth curves of $S$. aureus JE2 were done to ensure that exposure of the strain to $1 / 4 \times \mathrm{MIC}$ of either JBC $1847(0.25 \mathrm{mg} / \mathrm{L})$, T5 $(0.5 \mathrm{mg} / \mathrm{L})$, or promazine $(8 \mathrm{mg} / \mathrm{L})$ did not impair growth compared with the untreated control (Supplementary Figure 2).

An overnight culture of $S$. aureus JE2 grown on BA was prepared and a single colony was picked and grown in $\mathrm{MH}$ broth for 18-20 h. The overnight culture was diluted in BHI to $\mathrm{OD}_{600} 0.02$ and grown at $37^{\circ} \mathrm{C}$ with shaking, divided into four and treated with either $1 / 4 \times$ MIC of JBC $1847,1 / 4 \times$ MIC of $\mathrm{T} 5,1 / 4 \times \mathrm{MIC}$ of promazine, or left untreated for $30 \mathrm{~min}$ at $37^{\circ} \mathrm{C}$. The procedure was repeated three times, generating biological triplicates of the four treatment conditions. Total RNA from each sample was extracted applying the RNAeasy kit (Qiagen, Hilden, Germany) according to the manufacturer's instruction. The samples were stored at $-80^{\circ} \mathrm{C}$ until preparation for RNA sequencing.

\section{Library Preparation for RNA HiSeq Sequencing}

RNA concentrations of each of the 12 samples were measured using the Qubit BR RNA assay, and the RNA quality was evaluated using TapeStation with the RNA ScreenTape (Agilent Technologies, Santa Clara, CA, United States). All samples were rRNA depleted using the Ribo-Zero Plus rRNA Depletion Kit (Illumina, Inc., San Diego, CA, United States), and residual DNA from RNA extraction was removed using the DNase MAX kit (MoBio Laboratories Inc., Carlsbad, CA, United States). The samples were purified using the standard protocol for CleanPCR SPRI beads (CleanNA, Zuid, Netherlands) and further prepared for sequencing using the NEBNext Ultra II Directional RNA library preparation kit (New England Biolabs, MA, United States). Library concentrations were measured using Qubit HS DNA assay and library DNA size estimated using TapeStation with D1000 ScreenTape. The samples were pooled in equimolar concentrations and paired-end sequenced $(2 \times 150 \mathrm{bp})$ using a HiSEQ X platform (Illumina, United States). All kits were used as per the manufacturer's instructions with minor modifications.

\section{Transcriptome Mapping}

Forward and reverse DNA reads from the raw fastq files were filtered for PhiX using Bowtie 2 by aligning the reads against a database composed of the Coliphage phi-X174 genome (RefSeq accession NC_001422.1) indexed also using Bowtie2 (Langmead and Salzberg, 2012). Reads passing the PhiX-filtering were then trimmed for sequencing adaptors, quality filtered (Q-score > 20), and sequences shorter than 100 nucleotides discarded using Cutadapt (v2.10) (Marcel, 2013). Also using Cutadapt, sequencing adaptors were then trimmed from the reads passing the PhiX-filtering, and low-quality sequences (Q-score $\geq 15$ ) and those shorter than 100 nucleotides were discarded. A rRNA reference database was constructed by concatenating the small subunit (SSU) and large subunit (LSU) rRNA SILVA Parc databases and indexing using Bowtie2. Finally, reads were bioinformatically depleted for rRNA sequences by aligning reads against the constructed SSU/LSU SILVA Parc database using Bowtie2. The genome sequence of $S$. aureus JE2 with annotations (RefSeq accession GCF_002085525.1) was further annotated using Prokka (v1.14.6) (Seemann, 2014), and the resulting genome was indexed using Bowtie2. The rRNAdepleted and quality-filtered DNA reads were aligned against the genome with the Bowtie2-very-sensitive option, and all alignments were ported to unsorted .sam files. The .sam files from Bowtie2 were then sorted by sequence gene identifier (prokkaID) and converted to .bam files using samtools (v1.10). Finally, transcript/gene count tables were made using featureCounts (v2.0.1) (Li et al., 2009). Where nothing else is stated, the default settings were used for all tools. The DESeq 2 workflow was used to normalize read counts by the geometric mean count and identify differentially expressed genes. Importantly, rRNA gene expression levels were not included as both the RNA sample preparation and subsequent bioinformatic processing included rRNA depletion. Samples treated with either T5, JBC1847, 
or promazine were compared against the experiment control (Ctrl), and changes in gene transcript counts were considered statistically significant with the dual criteria of the observed fold change greater than twofold and the Benjamini-Hochberg corrected $p$-value $<0.05$.

\section{Differential Gene Expression Analysis}

The transcript/gene count tables were imported to RStudio ( $R$ Core Team, 2021) and processed using the default DESeq2 workflow and visualized using ggplot2. Principal component analysis (PCA) of overall sample similarity was done using the log-transformed, DESeq2 normalized counts.

\section{Metabolic Modulation}

For the functional annotation of the transcriptomes, the protein FASTA file of the translated CDS sequences (PROKKA_03112021.faa) was processed using the kyoto encyclopedia of genes and genomes (KEGG) Automatic Annotation Server Ver. 2.1 (KAAS, last update April 3, 2015) for automatic annotation and pathway reconstruction (Moriya et al., 2007). The bi-directional best hit (BBH) method was used to assign orthologs to sequences and the representative gene data set was set to prokaryotes. All other settings were left to default. The KEGG BRITE database (hierarchy file text), relating KO (KEGG Orthology) numbers with pathway information, was downloaded from https://www.kegg.jp/kegg-bin/get_htext?ko00001.keg (June 22, 2021) (Kanehisa and Goto, 2000; Kanehisa, 2019; Kanehisa et al., 2021). Files were cleaned retaining only the relationship between $\mathrm{KO}$ number and hierarchy level B. Barplots displaying the distribution of up- and downregulated genes per pathway were constructed using the predicted $\mathrm{KO}$ orthologs, the cleaned KEGG BRITE database, and identified differential expressed genes (DEGs) between conditions. All bioinformatic processing was done via RStudio IDE (1.2.1335) running $\mathrm{R}$ version 4.0.3 (October 10, 2020) (R Core Team, 2021) and using the R packages tidyverse (1.3.1) and ggplot2 (3.3.5).

\section{Macromolecule Biosynthesis}

To determine the possible impact of JBC 1847 on the synthesis rate of DNA, RNA, cell wall, and proteins, respectively, a macromolecule biosynthesis assay was conducted essentially according to Jana et al. (2017) with a modified protocol adapted to 96-well plates. Briefly, an overnight $S$. aureus JE2 culture was diluted 1:100 in Mueller Hinton Broth 2 (Millipore) and sub-cultured to $\mathrm{OD}_{600}$ of 0.2 . One milliliter of culture was distributed into each of four 1.5-ml Eppendorf tubes and the four radiolabeled precursors [ $(3 \mathrm{H})$-thymidine (Perkin Elmer), (3H)-uridine (Perkin Elmer), (3H)-isoleucine (American Radiolabeled Chemicals Inc.), and $(3 \mathrm{H})$-glucosamine hydrochloride (American Radiolabeled Chemicals Inc.)], respectively, were added to a concentration of $0.1 \mathrm{mCi} / \mathrm{ml}$. For each isotope, after vortexing, $100-\mu l$ volumes were immediately transferred to four microtiter wells to create two sets of samples with a technical replicate (two for JBC1847 and two for solvent control). To one pair of wells a concentration corresponding to $0.75 \times$ MIC of JBC 1847 was added and to a second pair of wells solvent was added corresponding to the concentration of solvent in the JBC 1847 samples. All wells were incubated/pulse labeled for $20 \mathrm{~min}$ at $37^{\circ} \mathrm{C}$. Subsequently, radiolabeled cells were precipitated with equal volume $(110 \mu \mathrm{l})$ of ice-cold $30 \%$ trichloroacetic acid (TCA) and left on ice for 1-2 h. Cell precipitates $(220 \mu \mathrm{l})$ were transferred to 96 -well format cellulose membrane filters (UniFilter GF/C, Perkin Elmer) using a FilterMate Harvester (Perkin Elmer), and were subsequently washed two times with $200 \mu \mathrm{l}$ ice-cold $15 \%$ TCA and one time with $200 \mu \mathrm{l}$ ice-cold water. The filter plate was dried overnight before $20 \mu \mathrm{l}$ of scintillation fluid was added to each well and $3 \mathrm{H}$ counts were obtained using a MicroBeta2 Microplate Reader (Perkin Elmer). Macromolecule biosynthesis rates were calculated based on the assumption that the rates of DNA, RNA, cell wall, and protein precursor incorporation were $100 \%$ in the control sample (treated with JBC 1847 solvent solution).

\section{Intracellular Adenosintrifosfat and Adenosine Diphosphate Measurements}

To investigate whether JBC 1847 affects the intracellular concentration of adenosintrifosfat (ATP) in S. aureus JE2 and/or the ATP/adenosine diphosphate (ADP) ratio, an ATP/ADP measurement assay was carried out. To measure intracellular ATP/ADP concentrations, JBC 1847 was initially dissolved in PBS to $40 \mathrm{mg} / \mathrm{L}$, which was further diluted to 2.5 and $10 \mathrm{mg} / \mathrm{L}$ solutions in PBS. S. aureus JE2 was inoculated in Mueller Hinton Broth II (MHB II) growth media and cultured overnight at $37^{\circ} \mathrm{C}$ with agitation (150 rpm). The overnight culture was sub-cultured at $1: 1,000$ dilution in $25 \mathrm{ml} \mathrm{MHB}$ II media and incubated at $37^{\circ} \mathrm{C}$ including agitation $(150 \mathrm{rpm})$, until reaching an $\mathrm{OD}_{600} 0.1$. The resulting $\mathrm{OD}_{600} 0.1$ culture was portioned in aliquots of $9 \mathrm{ml}$ in suitable vials and incubated for $4 \mathrm{~h}$. Subsequently, the prepared JBC 1847 solutions were added in appropriate volumes to yield final concentrations of $\mathrm{mg} / \mathrm{L} \mathrm{JBC} \mathrm{1847.} \mathrm{S.} \mathrm{aureus} \mathrm{incubated} \mathrm{with}$ blank PBS served as a negative control. Each treatment $(4 \mathrm{mg} / \mathrm{L}$ JBC 1847 or untreated negative control) was tested in triplicate $(n=3)$. The resulting mixtures were incubated $\left(37^{\circ} \mathrm{C}, 150 \mathrm{rpm}\right)$. Samples of $1 \mathrm{ml}$ were harvested after $t=15$ and $t=60 \mathrm{~min}$ of incubation. Samples were immediately centrifuged at 13,000 rpm for $10 \mathrm{~min}$ and the resulting pellet was re-suspended in MilliQ water. The resulting cell suspension was lysed by bead-beating and centrifuged at 13,000 rpm for $10 \mathrm{~min}$. The supernatant was analyzed using the ADP/ATP kit (ADP/ATP Ratio assay kit; Sigma-Aldrich) according to the manufacturer's instruction.

\section{Lysis of Erythrocytes}

The degree of compound-induced lysis of erythrocytes was investigated at concentrations up to $25 \mathrm{mg} / \mathrm{L}$ of JBC 1847 . Thus, JBC 1847 was dissolved in PBS to an initial concentration of $25 \mathrm{mg} / \mathrm{L}$ and treated with ultrasound. The initial stock solution was further diluted in PBS to produce solutions with concentrations of 1 and $5 \mathrm{mg} / \mathrm{L}$ and $300 \mu \mathrm{l}$ bovine whole blood was then mixed with $1,200 \mu \mathrm{l}$ sample solution. The mixture of whole blood and sample solution was agitated for $30 \mathrm{~min}$ by end-over-end rotation at ambient temperature. Subsequently, the mixture was centrifuged for $10 \mathrm{~min}$ at $13,000 \mathrm{rpm}$. The supernatant was diluted 20 -fold in ultrapure water and the absorbance of the diluted supernatant was measured at $540 \mathrm{~nm}$ (LabSystems Multiscanner plate reader). All samples were 
measured in duplicates. PBS and isotonic saline were included as negative controls where no lysis of red blood cells was expected. Ultrapure water and a solution of $0.1 \%$ Triton X-100 (AppliChem $\mathrm{GmbH}$, Darmstadt, Germany) in PBS were included as positive controls where complete lysis of erythrocytes was expected.

\section{RESULTS}

\section{Minimal Inhibitory Concentration, Minimal Bactericidal Concentration, and JBC 1847 Resistance Development}

The MIC of JBC 1847 was determined to equal $1 \mathrm{mg} / \mathrm{L}$ for both S. aureus JE2 and S. aureus CC398, and $2 \mathrm{mg} / \mathrm{L}$ for E. faecium. In all cases, culturing of $20 \mu \mathrm{l}$ from the wells corresponding to the MIC values revealed a $\mathrm{CFU} / \mathrm{ml}$ reduction of $\geq 99.9 \%$ compared with the initial inoculum in the wells. Thus, the MIC of JBC 1847 for these strains equaled the MBC. The MBC of JBC 1847 was equal to the $\mathrm{MBC}$ of exponential growing cells.

The MIC for both the DAP-sensitive IT1-S strain and the DAP-resistant variant equaled $1.5 \mathrm{mg} / \mathrm{L}$, indicating that DAP resistance does not cause cross-resistance to JBC 1847.

When investigating frequencies of single-step spontaneous mutations at plates containing either $4 \times \mathrm{MIC}(4 \mathrm{mg} / \mathrm{L})$ or $8 \times \mathrm{MIC}(8 \mathrm{mg} / \mathrm{L})$ of JBC 1847 , not a single S. aureus or E. faecium mutant could be found. Hence, the resistance frequency of single-step resistance-generating mutation(s) was $<7.33 \times 10^{-7}$, whereas the resistance frequencies for mupirocin and fusidic acid varied between $1.28 \times 10^{-4}$ and $2.82 \times 10^{-8}$ (Table 1), depending on the strain, antimicrobial, and concentration.

\section{JBC 1847 Dissipates the Cytoplasmic Membrane Proton Motive Force}

The depolarization effects of JBC 1847 on cell membrane potential depolarization were observed to be concentration dependent (Figure 1). CCCP was used as positive control for depolarization of the membrane. The same depolarizing effect can be observed when comparing $S$. aureus JE2 exposed to subinhibitory concentrations of JBC 1847 at $0.5 \mathrm{mg} / \mathrm{L}$ (1/2 MIC). These observations indicate that JBC 1847 strongly dissipates the cytoplasmatic membrane proton motive force (PMF).

\section{JBC 1847 Inhibits Macromolecule Biosynthesis}

The synthesis rates of all tested macromolecules (DNA, RNA, cell wall, and protein) were significantly decreased in $S$. aureus JE2 exposed to JBC 1847 for $20 \mathrm{~min}$ (Figure 2). In particular, the DNA and cell-wall synthesis rates were highly affected by JBC 1847 exposure.

\section{RNA Sequencing Results}

To investigate how JBC 1847 influences gene expression, the RNA expression profile was investigated in $S$. aureus JE2 exposed to sub-inhibitory concentrations of JBC 1847. The total number of raw DNA sequence reads (17-26 mio reads per sample) were nucleotide quality and phi-X filtered (generating between 14 


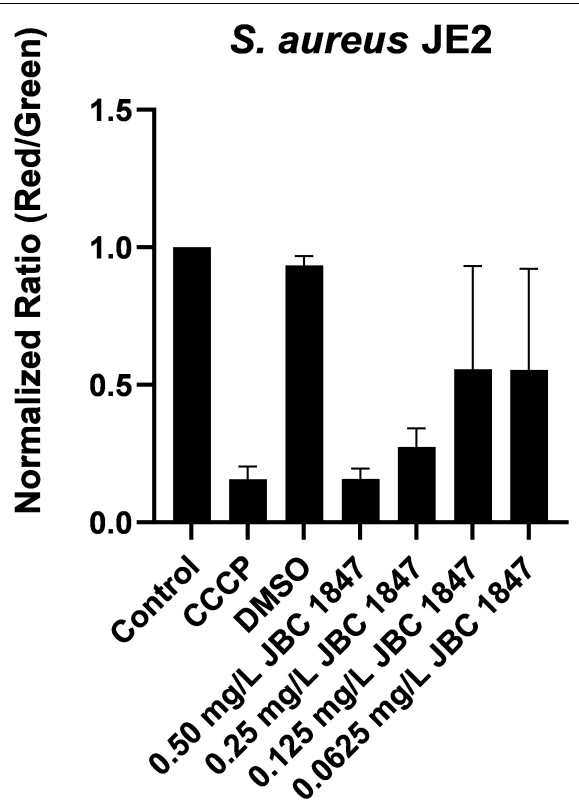

FIGURE 1 | Assessment of membrane potential using flow cytometry. Membrane depolarization assay showing the effect of $0.0625-0.50 \mathrm{mg} / \mathrm{L} \mathrm{JBC}$ 1847 on the membrane potential of Staphylococcus aureus JE2. Bar chart showing red/green mean fluorescence intensity ratio where a high ratio indicates high membrane potential and a low ratio a depolarized membrane. CCCP (2-[2-(3-chlorophenyl)hydrazinylyidene]propanedinitrile) is an oxidative phosphorylation uncoupler used as a positive control for membrane potential depolarization. Data are normalized against untreated control and show mean values of three biological replicates and SDs.

and 22 mio reads per sample) before mapping to the reference genome. On average, the ratio of the number of reads mapping to the reference compared with the number of quality filtered DNA reads was 0.993. Sample-wise PCA of the DESeq2-normalized gene expression data indicated that the samples readily cluster according to the experiment treatment groups (Figure 3). Figure 4 shows that all significantly regulated genes in JBC 1847 and T5 treated $S$. aureus were downregulated. Overall, the same genes were affected by T5 as JBC 1847 supporting the close approximation in the PCA plot of JBC 1847 and T5 treatments. In contrast, promazine-treated $S$. aureus exhibited a different expression profile where genes were both up- and downregulated (Figure 4). For JBC 1847 (and T5) treatments, genes related to the histidine biosynthesis and nitrate metabolism were the most abundant groups of regulated genes (Table 2). The RNA sequence data have been deposited in the Sequence Read Archive (SRA) from NCBI under the BioProject ID PRJNA766628.

\section{Kyoto Encyclopedia of Genes and Genomes Metabolic Modeling}

To further investigate the metabolic pathways of $S$. aureus treated with JBC 1847, a KEGG enrichment analysis of DEGs was performed. The DEGs were assigned to $12 \mathrm{KEGG}$ bioprocess pathways (Figure 5), of which all were downregulated compared with the untreated control. Genes belonging to signaling and cellular processes as well as amino acid metabolism were the most abundant (Figure 5). JBC 1847 exposure downregulated genes that are involved in histidine biosynthesis, nitrate reduction, and ATP-dependent bioprocesses (Table 2).

\section{JBC 1847 Decreases Intracellular Adenosintrifosfat and Increases the Adenosintrifosfat/Adenosine \\ Diphosphate Ratio}

To investigate if JBC 1847 influences the intracellular concentration of ATP and/or the ATP/ADP ratio in S. aureus

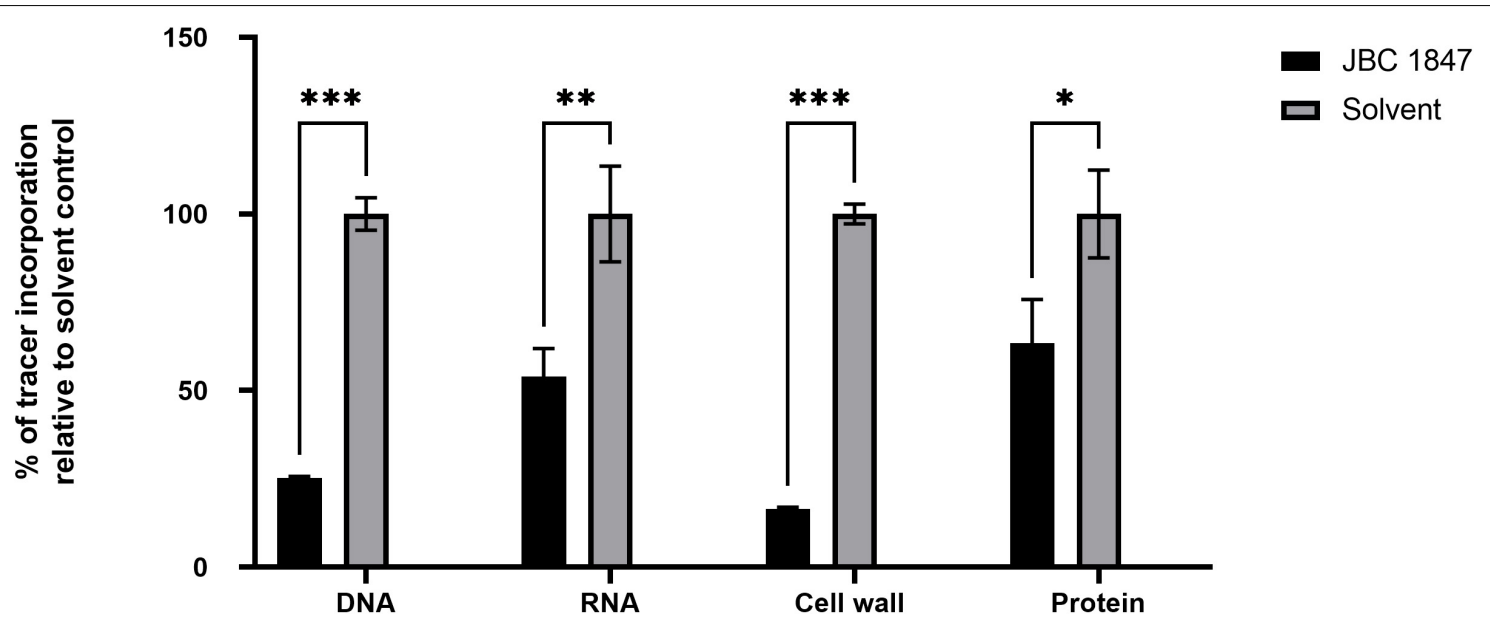

FIGURE 2 | Macromolecular synthesis assay in the presence of JBC 1847 and control (JBC 1847 solvent). Incorporation of radiolabeled precursors such as [3H] thymidine, $[3 \mathrm{H}]$ uridine, $[3 \mathrm{H}]$ leucine, $[14 \mathrm{C}] \mathrm{N}$-acetylglucosamine, and [3H] glycerol for DNA, RNA, protein, and cell wall synthesis, respectively, were quantified in Staphylococcus aureus JE2. Based on the incorporation of radiolabeled precursors, percent of inhibition by JBC 1847 was examined. Two biological replicates were used for each group and the statistical analysis was calculated by the two-tailed Student $t$-test. Each of the macromolecule groups were compared to the associated untreated control group. ${ }^{*} p<0.05 ;{ }^{\star *} p<0.01 ;{ }^{* \star *} p<0.001$. 


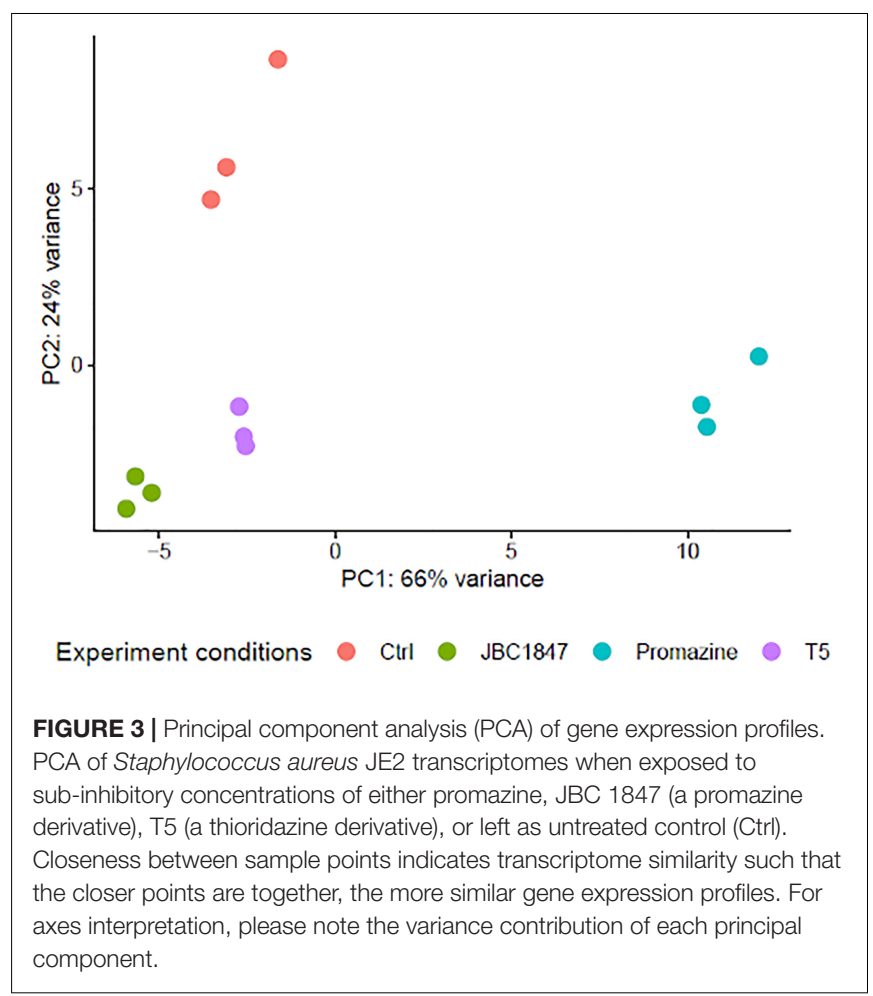

JE2, an ATP/ADP measurement assay was carried out. The results showed a marked reduction of intracellular ATP levels in $S$. aureus JE2 exposed to JBC 1847 for 15 min (Figure 6A). Compared with the PBS control, the ATP level remained significantly lower, even after $2 \mathrm{~h}$. In contrast, the ATP/APD ratio was significantly higher in cells exposed to JBC 1847 (Figure 6B).

\section{Erythrocyte Lysis}

Since JBC 1847 exhibits a cationic amphiphilic structure, its hemolytic activity was investigated. The lysis assay did not show any hemolytic activity of JBC 1847 evaluated in concentrations up to $25 \mathrm{mg} / \mathrm{L}$ (Figure 7), indicating JBC 1847 is at least partly selectively targeting the bacterial membrane of $S$. aureus.

\section{DISCUSSION}

Novel antimicrobials with new modes of action and low potential for bacterial resistance development are highly needed (Singer et al., 2019). Here, we have assessed the effect of JBC 1847, a potential novel antimicrobial, on $S$. aureus.

JBC 1847 is a cationic amphiphilic compound and has a quaternary ammonia structure and a constantly positive charge. The latter shares similarity to the very potent antimicrobial, daptomycin (DAP). DAP forms a positively charged complex with $\mathrm{Ca}^{++}$, which is a strict requirement for the activation of DAP (Grein et al., 2020). Both micelle formation of DAP and the positive charge of the $\mathrm{DAP}-\mathrm{Ca}^{++}$complex are assumed to contribute to the antimicrobial activity of DAP (Miller et al., 2016). However, in the present study we have shown that a DAP-resistant strain is not (cross-) resistant to JBC 1847; hence, the bacterial target(s) of JBC 1847 must be different from the target(s) of DAP. While the transcriptomic analysis revealed that a unique set of $S$. aureus genes were upregulated when exposed to sub-lethal concentrations of promazine, there was a complete lack of any upregulated genes in $S$. aureus JE2 in the presence of JBC 1847 (Figures 4A,C). Furthermore, the JBC 1847 exposed transcriptomes of S. aureus JE2 were strongly separated from the promazine transcriptomes in the PCA, indicating a highly different expression profile of $S$. aureus JE2, depending on treatment. Concordantly, we suggest that JBC 1847 has a different effect on S. aureus compared with its "mother" compound, promazine. The fact that $S$. aureus JE2 reacts differently to JBC 1847 exposure than to promazine exposure is not surprising since the MIC in $S$. aureus is considerably lower for JBC 1847 than for promazine. In this study, the MIC for JBC 1847 was found to be $1 \mathrm{mg} / \mathrm{L}$ whereas the MIC for promazine previously has been determined to be $128 \mathrm{mg} / \mathrm{L}$ in S. aureus JE2 (Jørgensen, 2020) and in other strains of $S$. aureus (Nehme et al., 2018). Furthermore, JBC 1847 is not acting synergistically with oxacillin whereas this property has been reported for various phenothiazines (Klitgaard et al., 2008; Hadji-Nejad et al., 2010) and previously we have shown via in silico analysis that JBC 1847 exhibits different pharmacological parameters than promazine (Ronco et al., $2020,2021 a)$. In contrast to promazine, the transcriptomic profile of $S$. aureus exposed to sub-inhibitory concentrations of JBC 1847 is highly similar to transcriptomic profile of $S$. aureus exposed to sub-inhibitory concentrations of T5, another phenothiazine [thioridazine (10-[2-(1-methylpiperidin2-yl)ethyl]-2-methylsulfanylphenothiazine) (Wassmann et al., 2018)] derivative (Jørgensen et al., 2020). This indicates that the structurally similar, positively charged side chains added to thioridazine as well as promazine to generate T5 and JBC 1847 , respectively, is of greater importance for the antimicrobial activity than which exact phenothiazine the derivatives originate from. For both T5 and JBC 1847, all DEGs were exclusively downregulated (Figure 4), with genes in pathways belonging to amino acid metabolism, nitrate reduction, and cell signaling as the most abundant DEGs (Table 2 and Figure 5). Among genes downregulated within amino acid metabolism, genes of the histidine metabolism were particularly abundant (Table 2). Downregulation of genes involved in the histidine metabolism has been shown to be crucial for cefquinomeinduced biofilm inhibition of Staphylococcus xylosus (Zhou et al., 2018). It is therefore likely that the strong biofilm-inhibiting properties of JBC 1847 previously documented in S. aureus, Staphylococcus epidermidis, and Cutibacterium acnes (Ronco et al., 2021b) are associated with JBC 1847-blocking of the histidine biosynthesis. In the same way, we have in the present study shown that JBC 1847 is active against bacteria in a slow metabolic state (stationary phase) characteristic of bacterial cell grown in biofilm. In addition to genes involved in the histidine biosynthesis, the two histidine kinase encoding genes, nraA and $n r e \mathrm{~B}$, were among the DEGs that were most downregulated by JBC 1847 . Recently, histidine kinase inhibitors have been suggested as novel antimicrobial drug targets due to their importance in various processes (Bem et al., 2015).

The finding of only downregulated DEGs in the transcriptome of JBC 1847 treated S. aureus indicates a "shut-down" of the 


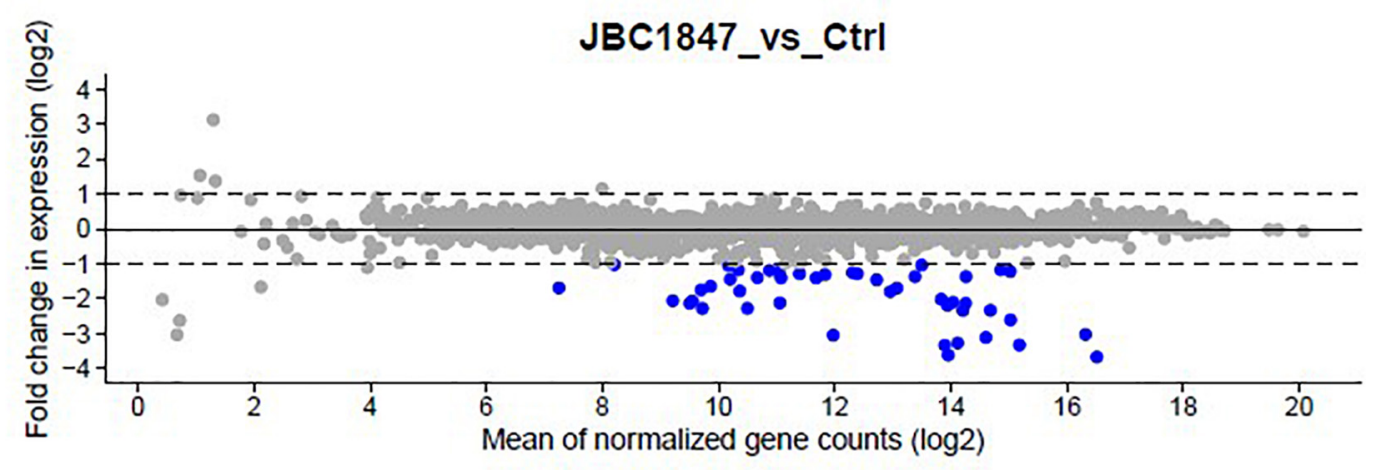

- Down: 48 - NS

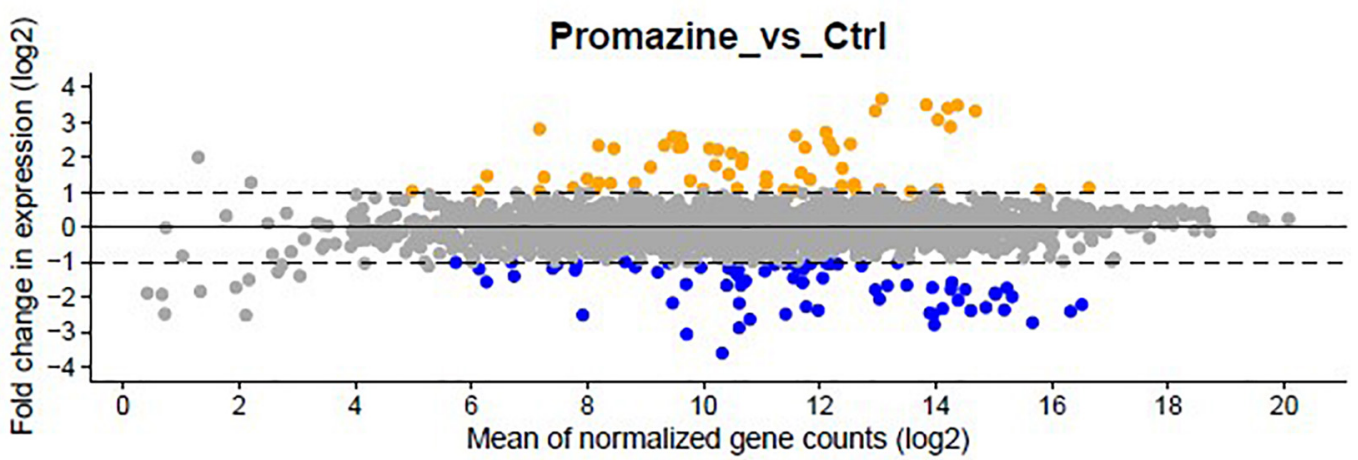

- Up: 59 - Down: 70 - NS

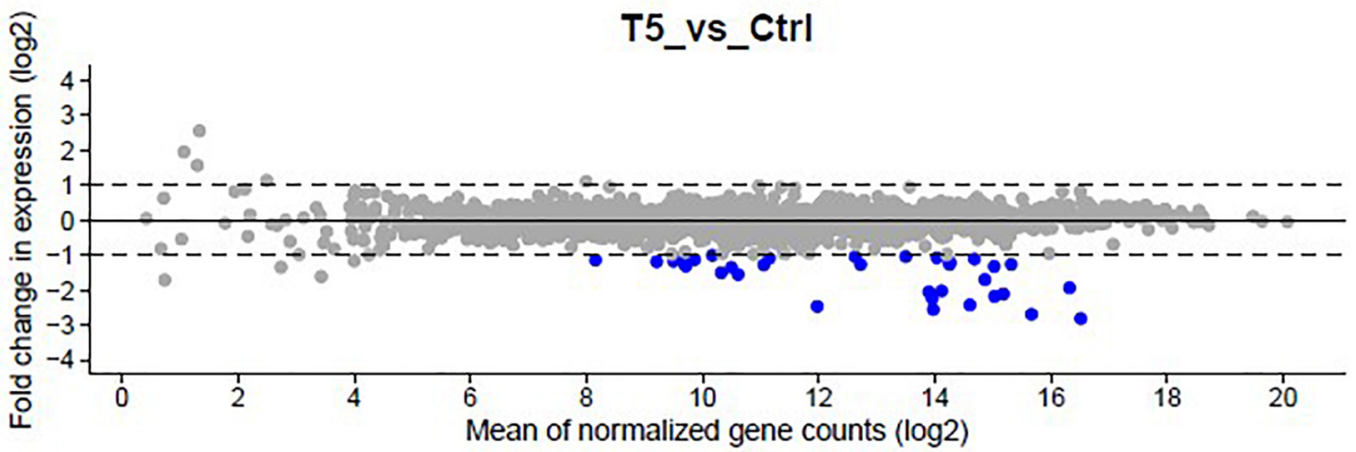

- Down: 35 - NS

FIGURE 4 | MA plots of gene expression in Staphylococcus aureus JE2. Visualization of gene expression of S. aureus exposed to JBC 1847 vs. untreated control (Ctrl) (upper figure), gene expression of S. aureus exposed to promazine vs. Ctrl (middle figure), and gene expression of S. aureus exposed to T5 vs. Ctrl (lower figure). The plot visualizes the differences between RNA levels in the two samples, by transforming the data onto M (log ratio) and A (mean average) scales, then plotting these values. Hence, the MA plots show the fold change in gene expression between experiment treatment groups vs. the untreated control (Ctrl) as a function of the averaged, normalized transcript count of each gene. Each point corresponds to one gene. Statistically significant up- and downregulated genes are shown in orange and blue, respectively. Non-significant (NS) changes are in gray. The axes are logarithmic (log2) and dashed lines indicate the $y$-axis levels corresponding to a twofold change in gene expression. Notice the complete lack of orange points in the upper and lower plots, indicating that none of the genes in S. aureus were upregulated by treatment with JBC 1847 or T5. In contrast, several genes were either up- or downregulated in promazine-treated S. aureus.

bacterial overall viability, which is supported by a significant inhibition in the synthesis rate of all investigated macromolecules (DNA, RNA, cell wall, and protein) (Figure 2).
As JBC 1847 led to complete depolarization of the cytoplasmic membrane (Figure 1), the PMF of the bacteria dissipates. PMF is the driving force for ATP synthesis, and in support of this, we 
TABLE 2 | The 30 genes with lowest p-values and highest 2 log-fold changes (downregulated) in Staphylococcus aureus JE2 exposed to 30 min of sub-inhibitory concentrations of JBC 1847 vs. untreated control conditions.

\begin{tabular}{|c|c|c|c|}
\hline Gene & Product & Fold change & $P$-value \\
\hline narH & Nitrate reductase subunit beta & -4.07 & $1.75 \times 10^{-148}$ \\
\hline narJ & Reductase molybdenum cofactor assembly chaperone & -4.02 & $1.23 \times 10^{-165}$ \\
\hline narG & Nitrate reductase subunit alpha & -3.66 & $4.96 \times 10^{-113}$ \\
\hline narl & Respiratory nitrate reductase subunit gamma & -3.61 & $9.14 \times 10^{-288}$ \\
\hline nreA & Respiration regulation sensor histidine kinase NreA & -3.32 & $2.89 \times 10^{-200}$ \\
\hline nreB & Nitrate respiration regulation sensor histidine kinase NreB & -3.32 & $1.10 \times 10^{-218}$ \\
\hline nreC & Nitrate respiration regulation response regulator NreC & -3.26 & $1.21 \times 10^{-259}$ \\
\hline cobA & Uroporphyrinogen-III C-methyltransferase & -3.10 & $1.64 \times 10^{-96}$ \\
\hline $\operatorname{nir} D$ & Nitrite reductase small subunit NirD & -3.04 & $6.0 \times 10^{-81}$ \\
\hline nark & NarK/NasA family nitrate transporter & -3.02 & $3.10 \times 10^{-174}$ \\
\hline nas $D$ & NAD(P)/FAD-dependent oxidoreductase & -2.60 & $2.91 \times 10^{-138}$ \\
\hline dapB & 4-Hydroxy-tetrahydrodipicolinate reductase & -2.33 & $1.92 \times 10^{-45}$ \\
\hline dapA & 4-Hydroxy-tetrahydrodipicolinate synthase & -2.33 & $2.57 \times 10^{-19}$ \\
\hline hisB & Imidazole glycerol-phosphate dehydratase HisB & -2.27 & $1.99 \times 10^{-36}$ \\
\hline hisC & Histidinol-phosphate aminotransferase family protein & -2.27 & $3.24 \times 10^{-44}$ \\
\hline hisH & Imidazole glycerol phosphate synthase subunit HisH & -2.13 & $1.22 \times 10^{-44}$ \\
\hline his $D$ & Histidinol dehydrogenase & -2.11 & $1.36 \times 10^{-35}$ \\
\hline hisA & Phosphoribosylformimino-5-aminoimidazole carboxamide ribotide isomerase & -2.06 & $7.15 \times 10^{-35}$ \\
\hline hisZ & Adenosintrifosfat (ATP) phosphoribosyltransferase & -2.05 & $1.12 \times 10^{-35}$ \\
\hline hisE & Bifunctional phosphoribosyl-AMP Cyclohydrolase/phosphoribosyl-ATP diphosphatase HisIE & -1.77 & $2.32 \times 10^{-38}$ \\
\hline hisF & Imidazole glycerol phosphate synthase subunit HisF & -1.74 & $7.73 \times 10^{-35}$ \\
\hline dapD & 2,3,4,5-Tetrahydropyridine-2,6-dicarboxylate $\mathrm{N}$-acetyltransferase & -2.01 & $2.50 \times 10^{-82}$ \\
\hline$n d h F$ & NADH dehydrogenase subunit 5 & -1.6 & $2.20 \times 10^{-21}$ \\
\hline$i l v C$ & Ketol-acid reductoisomerase & -1.44 & $6.26 \times 10^{-25}$ \\
\hline$i l v B$ & Biosynthetic-type acetolactate synthase large subunit & -1.40 & $4.66 \times 10^{-18}$ \\
\hline$i l v D$ & Dihydroxy-acid dehydratase & -1.22 & $2.82 \times 10^{-11}$ \\
\hline leuA & 2-Isopropylmalate synthase & -1.39 & $1.26 \times 10^{-21}$ \\
\hline YbcC & YbcC family protein & -1.37 & $3.70 \times 10^{-26}$ \\
\hline $\operatorname{argH}$ & Argininosuccinate lyase & -1.36 & $3.39 \times 10^{-22}$ \\
\hline $\arg G$ & Argininosuccinate synthase & -1.25 & $6.42 \times 10^{-40}$ \\
\hline $\operatorname{esc} A$ & ATP-binding cassette domain-containing protein & -1.40 & $4.22 \times 10^{-12}$ \\
\hline asd & Aspartate-semialdehyde dehydrogenase & -2.10 & $2.20 \times 10^{-43}$ \\
\hline$m n h$ & $\mathrm{Na}^{+} / \mathrm{H}^{+}$antiporter family protein & -1.22 & $7.70 \times 10^{-31}$ \\
\hline rlp & Peptide ABC transporter substrate-binding protein & -1.28 & $7.15 \times 10^{-18}$ \\
\hline $\operatorname{lrg} A$ & Antiholin-like murein hydrolase modulator LrgA & -1.17 & $5.98 \times 10^{-6}$ \\
\hline cbix & Sirohydrochlorin chelatase & -2.18 & $1.82 \times 10^{-35}$ \\
\hline
\end{tabular}

No significantly upregulated genes were identified in the same transcriptomic analysis.

found that JBC 1847 led to a marked decrease in intracellular ATP (Figure 6). Since the membrane potential, PMF, and intracellular ATP level influence $\mathrm{pH}$ homeostasis, membrane transport, motility cell division, bacterial communication, and environmental sensing (Benarroch and Asally, 2020), JBC 1847 targets many vital bacterial processes. A correlation between antibiotic-induced decreased intracellular ATP level and increased killing of bacteria has been shown in Mycobacteria tuberculosis (Rao et al., 2008), which is in agreement with previous reports on significant antimicrobial effect of phenothiazines on M. tuberculosis in particular (Amaral and Molnar, 2010). The downregulation of numerous genes in the histidine metabolism observed in this study could also be a result of limited ATP, as the initial step of the histidine biosynthesis is ATP dependent (Ohta et al., 2000). It is therefore not possible to differentiate if downregulated DEGs of the histidine biosynthesis is a primary cause of JBC 1847 exposure or secondary to the depletion of cellular ATP. Nevertheless, both the inhibition of histidine biosynthesis pathway (Lunardi et al., 2013) and inhibition of the ATP synthase (Liu et al., 2020) have been suggested as attractive targets for development of novel antimicrobials.

Whereas the ATP level was markedly decreased, there was an increase in the ATP/ADP ratio in JBC 1847-treated cells. A similar rise in the ATP/ADP ratio has been observed in Escherichia coli exposed to antibiotics, in which it was concluded that (relative) ATP accumulation was not a result of increased respiration, but was due to inhibition of energy-consuming processes (Akhova and Tkachenko, 2014). This is in accordance with the observations on decreased synthesis of macromolecules in our study, processes all depending on energy. In addition, other studies have found that a high ATP/ADP ratio limits the 


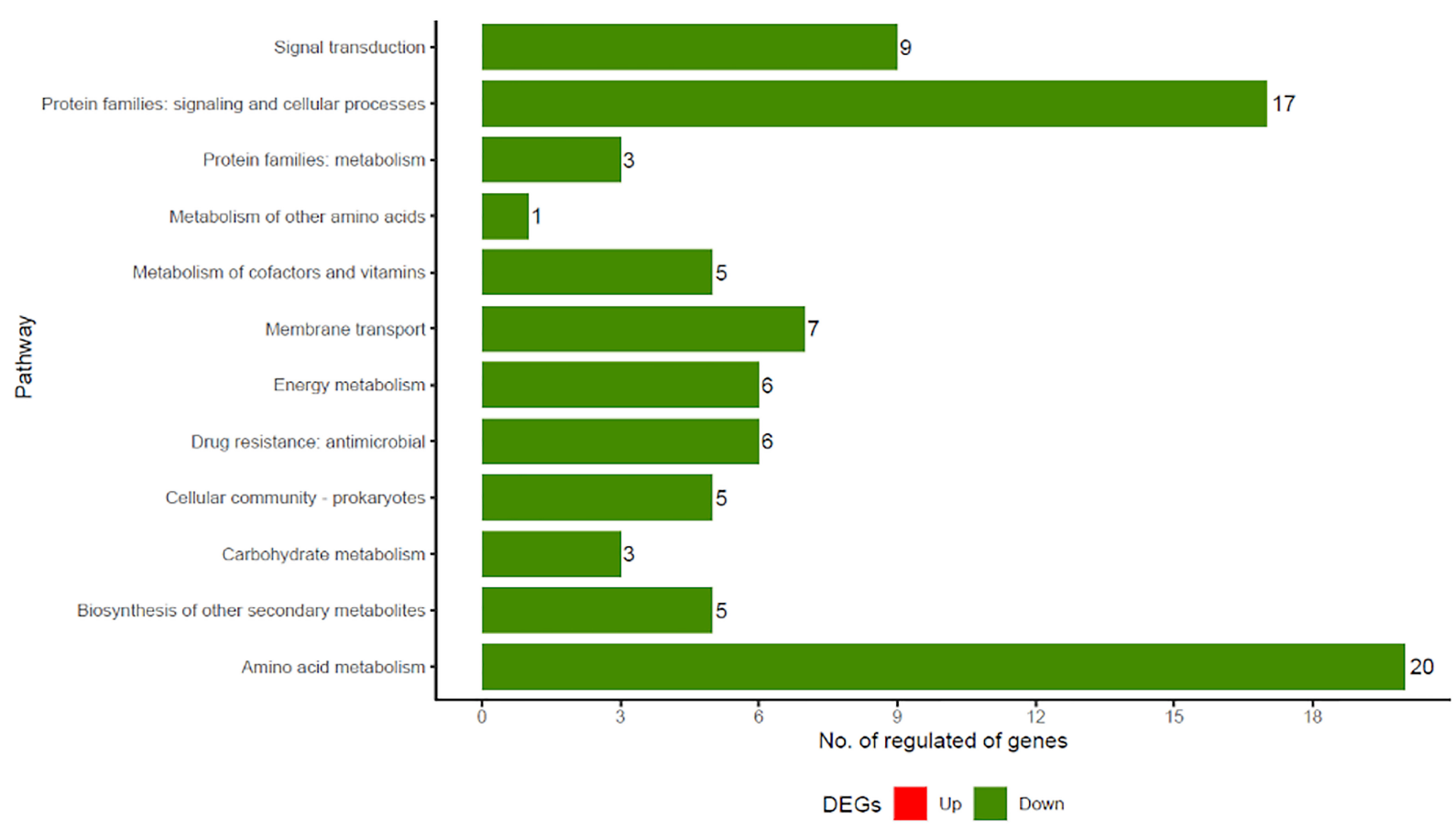

FIGURE 5 | Kyoto encyclopedia of genes and genomes (KEGG) functional classification of regulated genes in Staphylococcus aureus JE2 exposed to JBC 1847. Only genes significantly differently expressed from genes in the untreated control are included. Note the absence of red bars (upregulated genes) among the differentially expressed genes (DEGs). The number of genes assigned to each pathway is stated to the right of the bars.

\section{A}

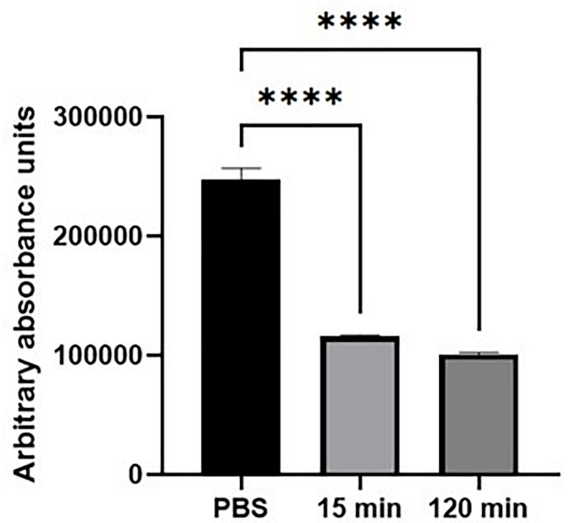

B

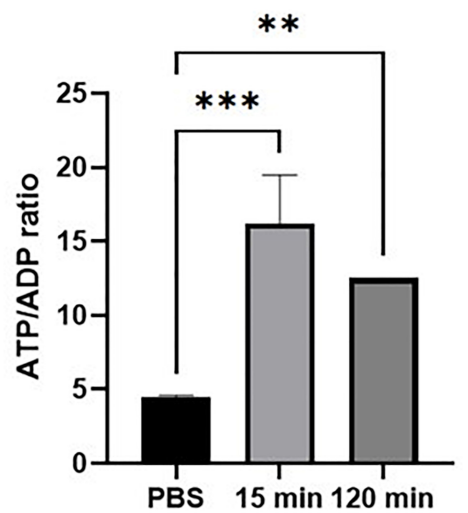

FIGURE 6 | Intracellular adenosintrifosfat (ATP) level (measured in absorbance units) (A) and ATP/adenosine diphosphate (ADP) ratio (B) in Staphylococcus aureus after exposure to JBC 1847 (4 mg/L) in either 15 or $120 \mathrm{~min}$. The error bars show SD of three replicates. ${ }^{\star \star} p<0.01 ;{ }^{* \star \star} p<0.001 ;{ }^{{ }^{* \star \star *}} p<0.0001$.

ATP synthesis rate (Meyrat and von Ballmoos, 2019), which could also contribute to a lowered cellular concentration of ATP, in accordance with the results of the present study (Figure 7). The uncoupling of the cellular respiration and, hence, lower level of ATP has been associated with a potentiation of bactericidal antibiotics (Lobritz et al., 2015) which is characteristic for kinetics of JBC 1847. Bedaquiline, an antibiotic used for treatment of tuberculosis, also targets ATP synthase, uncoupling the cellular respiration from ATP synthesis, and as consequence, a futile proton cycle that is linked to cell death is induced (Hards et al., 2015).

In the lack of oxygen, nitrate can serve as anaerobic electron acceptor in the electron chain of $S$. aureus (Burke and Lascelles, 1975); hence, it aids in extrusion of protons from the cellular cytoplasm to generate a PMF (Chen and Strous, 2013). In addition, nitrate reduction can also be coupled to the fermentation of organic acids such as formate to allow for survival in the presence of compounds that dissipate PMF. Therefore, it 


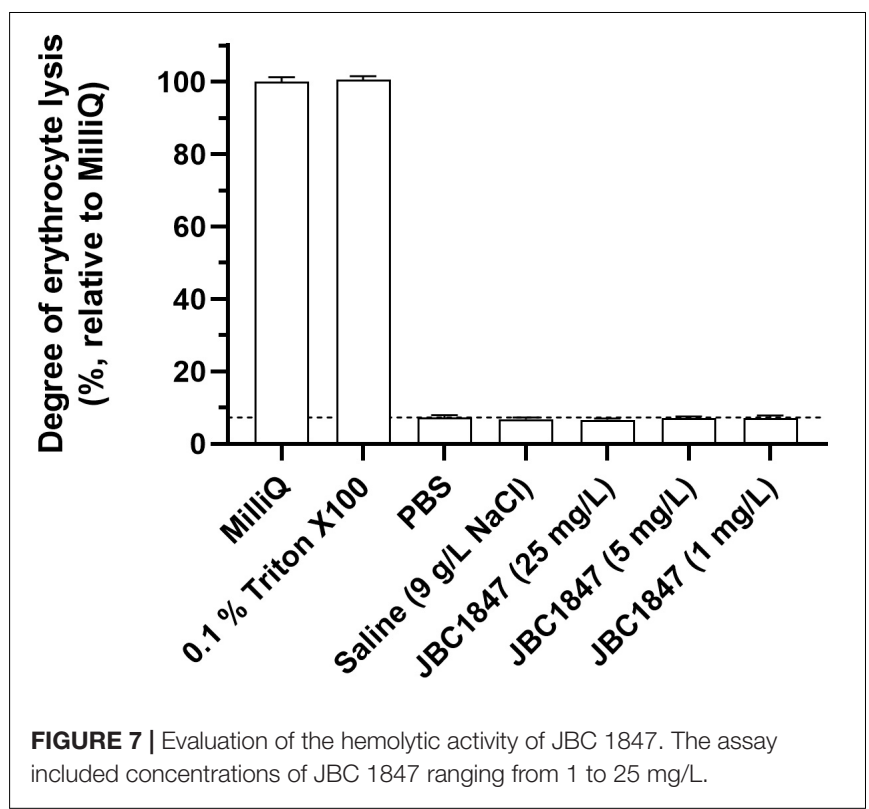

was somewhat surprising to find that genes essential for nitrate reductase were among the most downregulated. In $S$. aureus exposed to nisin, another membrane depolarization compound, genes encoding nitrate and nitrate reductase were up to 12 times upregulated (Muthaiyan et al., 2008), most likely to compensate for the diminished PMF. In contrast, our results indicate that JBC 1847 inhibits the use of nitrate as an alternative electron acceptor, hence, is not able to counteract the diminished PMF by this pathway.

For the spontaneous mutation assay, we were not able to detect a single $S$. aureus or E. faecium isolate with increased tolerance to JBC 1847, in contrast to the relatively high resistance frequencies of the same species against fusidic acid and, to a lesser extent, mupirocin (Table 1). The finding of these low spontaneous resistance frequencies of JBC 1847 is somewhat surprising, as bacterial resistance to uncouplers have been described numerous times for both Gram-positive and Gramnegative bacteria (Krulwich et al., 1990; Lewis et al., 1994; Čubopová et al., 2004). However, the multitarget activity of JBC 1847 as proposed by the results in the present study (uncoupling effect combined with inhibition of the histidine biosynthesis and nitrate reductase pathways) seems difficult for the bacteria to counteract with an increased tolerance response, as shown in the present study for single-step mutations and in a previous study using an induced tolerance (daily sub-culturing, low concentrations) assay (Ronco et al., 2020).

\section{CONCLUSION}

Multidrug-resistant pathogens infecting humans and animals constitute a serious One Health issue worldwide and, therefore, there is a need for novel antimicrobial treatment solutions. Here, we have investigated the effect of JBC 1847, a potential new antimicrobial compound, on $S$. aureus. We have shown that JBC 1847 causes depolarization of the cell membrane resulting in dissipation of the proton motive (PMF), whereby many essential bacterial processes are affected. Concordantly, JBC 1847 caused a lowered intracellular level of ATP, decreased the synthesis of macromolecules (DNA, RNA, cell wall, and protein) and mediated downregulation of genes essential for the amino acid metabolism. Since JBC 1847 seems to target essential, non-redundant bacterial mechanisms, compensatory mechanisms seem to be limited supported by the observed low rate of resistance toward the compounds. Future studies are needed to verify that the bacterial impact of JBC 1847 suggested in the present study is corresponding to the activity of the compound in in vivo settings, and further addressing the degree of JBC 1847 eukaryotic cytotoxicity.

\section{DATA AVAILABILITY STATEMENT}

The datasets presented in this study can be found in online repositories. The names of the repository/repositories and accession number(s) can be found below: https://www.ncbi.nlm. nih.gov/genbank/, NC_001422.1.

\section{AUTHOR CONTRIBUTIONS}

AS, MM, VA, LK, NB, SS, LS, MA, and AF: experimental work. TR, LS, and KH: statistics, figures, and tables. TR, LK, JC, and RO: validation of results. TR and RO: wrote the manuscript. TR, $\mathrm{AP}, \mathrm{RO}, \mathrm{KH}, \mathrm{AP}$, and JK: manuscript review. JC, RO, AS, and BJ: experimental design. All authors contributed to the article and approved the submitted version.

\section{FUNDING}

This study was supported financially by a grant from the Novo Nordisk Foundation (NNF20OC0066116) and a grant from Innovation Fund Denmark (9122-0074B).

\section{ACKNOWLEDGMENTS}

We would like to thank DNAsense for skillful HiSeq sequencing of the $S$. aureus transcriptomes.

\section{SUPPLEMENTARY MATERIAL}

The Supplementary Material for this article can be found online at: https://www.frontiersin.org/articles/10.3389/fmicb.2021. 786173/full\#supplementary-material

Supplementary Figure 1 | Molecular structure of JBC 1847 (A), T5 (B), and promazine (C).

Supplementary Figure 2 | CFU/ml of Staphylococcus aureus JE2 exposed to 1/4 MIC of either JBC 1847 (0.25 mg/L), T5 (0.5 mg/L), promazine (8 mg/L), or left untreated. 


\section{REFERENCES}

Akhova, A. V., and Tkachenko, A. G. (2014). ATP/ADP alteration as a sign of the oxidative stress development in Escherichia coli cells under antibiotic treatment. FEMS Microbiol. Lett. 353, 69-76. doi: 10.1111/1574-6968.12405

Amaral, L., and Molnar, J. (2010). Therapy of XDR TB with thioridazine a drug beyond patent protection but eligible for patent "As New Use". Recent Pat. Antiinfect. Drug Discov. 5, 109-114. doi: 10.2174/157489110791233540

Anderl, J. N., Zahller, J., Roe, F., and Stewart, P. S. (2003). Role of nutrient limitation and stationary-phase existence in Klebsiella pneumoniae biofilm resistance to ampicillin and ciprofloxacin. Antimicrob. Agents Chemother. 47, 1251-1256. doi: 10.1128/AAC.47.4.1251-1256.2003

Bem, A. E., Velikova, N., Pellicer, M. T., Baarlen, P., Van Marina, A., and Wells, J. M. (2015). Bacterial histidine kinases as novel antibacterial drug targets. ACS Chem. Biol. 10, 213-224. doi: 10.1021/cb5007135

Benarroch, J. M., and Asally, M. (2020). The microbiologist's guide to membrane potential dynamics. Trends Microbiol. 28, 304-314. doi: 10.1016/j.tim.2019.12. 008

Burke, K. A., and Lascelles, J. (1975). Nitrate reductase system in Staphylococcus aureus wild type and mutants. J. Bacteriol. 123, 308-316. doi: 10.1128/jb.123.1. 308-316.1975

Butler, M. M., Skow, D. J., Stephenson, R. O., Lyden, P. T., LaMarr, W. A., and Foster, K. A. (2002). Low frequencies of resistance among Staphylococcus and Enterococcus species to the bactericidal DNA polymerase inhibitor N3-hydroxybutyl 6-(3'-ethyl-4'-methylanilino) uracil. Antimicrob. Agents Chemother. 46, 3770-3775. doi: 10.1128/AAC.46.12.3770-3775. 2002

Cassini, A., Högberg, L. D., Plachouras, D., Quattrocchi, A., Hoxha, A., Simonsen, G. S., et al. (2019). Attributable deaths and disability-adjusted life-years caused by infections with antibiotic-resistant bacteria in the EU and the European Economic Area in 2015: a population-level modelling analysis. Lancet Infect. Dis. 19, 56-66. doi: 10.1016/S1473-3099(18)30605-4

Chen, J., and Strous, M. (2013). Denitrification and aerobic respiration, hybrid electron transport chains and co-evolution. Biochim. Biophys. Acta Bioenerg. 1827, 136-144. doi: 10.1016/j.bbabio.2012.10.002

Čubopová, L., Šurín, S., Majerník, A., and Šmigáň, P. (2004). Isolation and characterization of an uncoupler-resistant mutant of Methanothermobacter thermautotrophicus. FEMS Microbiol. Lett. 233, 23-28. doi: 10.1016/j.femsle. 2004.01.033

Fey, P. D., Endres, J. L., Yajjala, V. K., Yajjala, K., Widhelm, T. J., Boissy, R. J., et al. (2013). A genetic resource for rapid and comprehensive phenotype screening of nonessential Staphylococcus aureus genes. mBio 4:e0537-12. doi: 10.1128/mBio. 00537-12.Editor

Grein, F., Müller, A., Scherer, K. M., Liu, X., Ludwig, K. C., Klöckner, A., et al. (2020). $\mathrm{Ca}^{2+}$-Daptomycin targets cell wall biosynthesis by forming a tripartite complex with undecaprenyl-coupled intermediates and membrane lipids. Nat. Commun. 11:1455. doi: 10.1038/s41467-020-15257-1

Hadji-Nejad, S., Rahbar, M., and Mehrgan, H. (2010). Synergy between phenothiazines and oxacillin against clinical isolates of methicillin-resistant Staphylococcus aureus. Trop. J. Pharm. Res. 9, 243-249. doi: 10.4314/tjpr.v9i3. 56284

Hards, K., Robson, J. R., Berney, M., Shaw, L., Bald, D., Koul, A., et al. (2015). Bactericidal mode of action of bedaquiline. J. Antimicrob. Chemother. 70, 2028-2037. doi: 10.1093/jac/dkv054

Jana, B., Baker, K. R., and Guardabassi, L. (2017). Macromolecule biosynthesis assay and fluorescence mode(s) of action. Antimicrob. Pept. Methods Protoc. 1548, 181-190. doi: 10.1007/978-1-4939-6737-7_12

Jørgensen, N. S. (2020). An Evaluation of the Antimicrobial Activity of a Novel Synthetic Compound. Master thesis. Copenhagen: University of Copenhagen.

Jørgensen, N. S., Saaby, L., Andersson, A. M., Kromann, S., Sheikhsamani, E., Permin, A., et al. (2020). A novel derivative of thioridazine shows low toxicity and efficient activity against gram-positive pathogens. Antibiotics 9:327. doi: 10.3390/antibiotics 9060327

Kanehisa, M. (2019). Toward understanding the origin and evolution of cellular organisms. Protein Sci. 28, 1947-1951. doi: 10.1002/pro.3715

Kanehisa, M., Furumichi, M., Sato, Y., Ishiguro-Watanabe, M., and Tanabe, M. (2021). KEGG: integrating viruses and cellular organisms. Nucleic Acids Res. 49, D545-D551. doi: 10.1093/nar/gkaa970
Kanehisa, M., and Goto, S. (2000). KEGG: kyoto encyclopedia of genes and genomes. Nucleic Acids Res. 28, 27-30.

Klitgaard, J. K., Skov, M. N., Kallipolitis, B. H., and Kolmos, H. J. (2008). Reversal of methicillin resistance in Staphylococcus aureus by thioridazine. J. Antimicrob. Chemother. 62, 1215-1221. doi: 10.1093/jac/dkn417

Kmietowicz, Z. (2017). Few novel antibiotics in the pipeline, WHO warns. BMJ 358:j4339. doi: 10.1136/bmj.j4339

Kristiansen, J. E., Hendricks, O., Delvin, T., Butterworth, T. S., Aagaard, L., Christensen, J. B., et al. (2007). Reversal of resistance in microorganisms by help of non-antibiotics. J. Antimicrob. Chemother. 59, 1271-1279. doi: 10.1093/jac/ dkm071

Krulwich, T. A., Quirk, P. G., and Guffanti, A. A. (1990). Uncoupler-resistant mutants of bacteria. Microbiol. Rev. 54, 52-65. doi: 10.1128/mmbr.54.1.52-65. 1990

Langmead, B., and Salzberg, S. L. (2012). Fast gapped-read alignment with Bowtie 2. Nat. Methods 9, 357-359. doi: 10.1038/nmeth.1923

Lewis, K., Naroditskaya, V., Ferrante, A., and Fokina, I. (1994). Bacterial resistance to uncouplers. J. Bioenerg. Biomembr. 26, 639-646. doi: 10.1007/BF00831539

Li, H., Handsaker, B., Wysoker, A., Fennell, T., Ruan, J., Homer, N., et al. (2009). The sequence alignment/map format and SAMtools. Bioinformatics 25, 20782079. doi: 10.1093/bioinformatics/btp352

Liu, L., Beck, C., Nøhr-Meldgaard, K., Peschel, A., Kretschmer, D., Ingmer, H., et al. (2020). Inhibition of the ATP synthase sensitizes Staphylococcus aureus towards human antimicrobial peptides. Sci. Rep. 10:11391. doi: 10.1038/s41598020-68146-4

Lobritz, M. A., Belenky, P., Porter, C. B. M., Gutierrez, A., Yang, J. H., Schwarz, E. G., et al. (2015). Antibiotic efficacy is linked to bacterial cellular respiration. Proc. Natl. Acad. Sci. U.S.A. 112, 8173-8180. doi: 10.1073/pnas.1509743112

Lunardi, J., Nunes, J. E. S., Bizarro, C. V., Basso, L. A., Santos, D. S., and Machado, P. (2013). Targeting the Histidine pathway in Mycobacterium tuberculosis. Curr. Top. Med. Chem. 13, 2866-2884. doi: 10.2174/15680266113136660203

Marcel, M. (2013). Cutadapt removes adapter sequences from high-throughput sequencing reads. EMBnet J. 17, 10-12. doi: 10.1089/cmb.2017.0096

Meyrat, A., and von Ballmoos, C. (2019). ATP synthesis at physiological nucleotide concentrations. Sci. Rep. 9:3070. doi: 10.1038/s41598-019-38564-0

Miller, W. R., Bayer, A. S., and Arias, C. A. (2016). Mechanism of action and resistance to daptomycin in Staphylococcus aureus and Enterococci. Cold Spring Harb. Perspect. Med. 6:a026997. doi: 10.1101/cshperspect.a026997

Moriya, Y., Itoh, M., Okuda, S., Yoshizawa, A. C., and Kanehisa, M. (2007). KAAS: an automatic genome annotation and pathway reconstruction server. Nucleic Acids Res. 35, 182-185. doi: 10.1093/nar/gkm321

Muthaiyan, A., Silverman, J. A., Jayaswal, R. K., and Wilkinson, B. J. (2008). Transcriptional profiling reveals that daptomycin induces the Staphylococcus aureus cell wall stress stimulon and genes responsive to membrane depolarization. Antimicrob. Agents Chemother. 52, 980-990. doi: 10.1128/AAC. 01121-07

Nehme, H., Saulnier, P., Ramadan, A. A., Cassisa, V., Guillet, C., Eveillard, M., et al. (2018). Antibacterial activity of antipsychotic agents, their association with lipid nanocapsules and its impact on the properties of the nanocarriers and on antibacterial activity. PLoS One 13:e0189950. doi: 10.1371/journal.pone. 0189950

Ohta, D., Fujimori, K., Mizutani, M., Nakayama, Y., Kunpaisal-Hashimoto, R., Munzer, S., et al. (2000). Molecular cloning and characterization of ATPphosphoribosyl transferase from Arabidopsis, a key enzyme in the histidine biosynthetic pathway. Plant Physiol. 122, 907-914. doi: 10.1104/pp.122.3.907

Price, L. B., Stegger, M., Hasman, H., Aziz, M., Larsen, J., Andersen, S., et al. (2012). Staphylococcus aureus CC398: host adaptation and emergence of methicillin resistance in livestock. mBio 3:e0305-11. doi: 10.1128/mBio.00305-11

R Core Team (2021). R: A Language and Environment for Statistical Computing. Vienna: R Foundation for Statistical Computing.

Rao, S. P. S., Alonso, S., Rand, L., Dick, T., and Pethe, K. (2008). The protonmotive force is required for maintaining ATP homeostasis and viability of hypoxic, nonreplicating Mycobacterium tuberculosis. Proc. Natl. Acad. Sci. U.S.A. 105, 11945-11950. doi: 10.1073/pnas.0711697105

Ronco, T., Aragao, F. M., Saaby, L., Christensen, J. B., Permin, A., Williams, A. R., et al. (2021a). A new phenothiazine derivate is active against Clostridioides difficile and shows low cytotoxicity. PLoS One 16:e258207. doi: 10.1371/ journal.pone.0258207 
Ronco, T., Aragao, M. F., Svenningsen, S., Christensen, J. B., Permin, A., Saaby, L., et al. (2021b). Efficacy of a novel antimicrobial hydrogel for eradication of Staphylococcus epidermidis, Staphylococcus aureus and Cutibacterium acnes from preformed biofilm and treatment performance in an in vivo MRSA wound model. JAC Antimicrob. Resist. 3:dlab108. doi: 10.1093/jacamr/ dlab108

Ronco, T., Jørgensen, N. S., Holmer, I., Kromann, S., Sheikhsamani, E., Permin, A., et al. (2020). A novel promazine derivative shows high in vitro and in vivo antimicrobial activity against Staphylococcus aureus. Front. Microbiol. 11:560798. doi: 10.3389/fmicb.2020.560798

Sabat, A. J., Tinelli, M., Grundmann, H., Akkerboom, V., Monaco, M., Del Grosso, M., et al. (2018). Daptomycin resistant staphylococcus aureus clinical strain with novel non-synonymous mutations in the $m p r F$ and vraS genes: a new insight into daptomycin resistance. Front. Microbiol. 9:2705. doi: 10.3389/fmicb.2018. 02705

Seemann, T. (2014). Prokka: rapid prokaryotic genome annotation. Bioinformatics 30, 2068-2069. doi: 10.1093/bioinformatics/btu153

Sharma, D., Misba, L., and Khan, A. U. (2019). Antibiotics versus biofilm: an emerging battleground in microbial communities. Antimicrob. Resist. Infect. Control 8:76. doi: 10.1186/s13756-019-0533-3

Singer, A. C., Kirchhelle, C., and Roberts, A. P. (2019). Reinventing the antimicrobial pipeline in response to the global crisis of antimicrobial-resistant infections [version 1; referees: 2 approved]. F1000Research 8:238. doi: 10.12688/ f1000research.18302.1

Stenger, M., Behr-Rasmussen, C., Klein, K., Grønnemose, R. B., Andersen, T. E., Klitgaard, J. K., et al. (2017). Systemic thioridazine in combination with dicloxacillin against early aortic graft infections caused by Staphylococcus aureus in a porcine model: in vivo results do not reproduce the in vitro synergistic activity. PLoS One 12:e0173362. doi: 10.1371/journal.pone.017 3362
Wassmann, C. S., Lund, L. C., Thorsing, M., Lauritzen, S. P., Kolmos, H. J., Kallipolitis, B. H., et al. (2018). Molecular mechanisms of thioridazine resistance in Staphylococcus aureus. PLoS One 13:e0201767. doi: 10.1371/journal.pone. 0201767

Zhou, Y. H., Xu, C. G., Yang, Y. B., Xing, X. X., Liu, X., Qu, Q. W., et al. (2018). Histidine metabolism and IGPD play a key role in cefquinome inhibiting biofilm formation of Staphylococcus xylosus. Front. Microbiol. 9:665. doi: $10.3389 /$ fmicb. 2018.00665

Conflict of Interest: AP was employed by company Unibrains. LS and $\mathrm{KH}$ were employed by company Bioneer A/S.

The remaining authors declare that the research was conducted in the absence of any commercial or financial relationships that could be construed as a potential conflict of interest.

Publisher's Note: All claims expressed in this article are solely those of the authors and do not necessarily represent those of their affiliated organizations, or those of the publisher, the editors and the reviewers. Any product that may be evaluated in this article, or claim that may be made by its manufacturer, is not guaranteed or endorsed by the publisher.

Copyright (C) 2022 Ronco, Kappel, Aragao, Biagi, Svenningsen, Christensen, Permin Saaby, Holmstrøm, Klitgaard, Sabat, Akkerboom, Monaco, Tinelli, Friedrich, Jana and Olsen. This is an open-access article distributed under the terms of the Creative Commons Attribution License (CC BY). The use, distribution or reproduction in other forums is permitted, provided the original author(s) and the copyright owner(s) are credited and that the original publication in this journal is cited, in accordance with accepted academic practice. No use, distribution or reproduction is permitted which does not comply with these terms. 